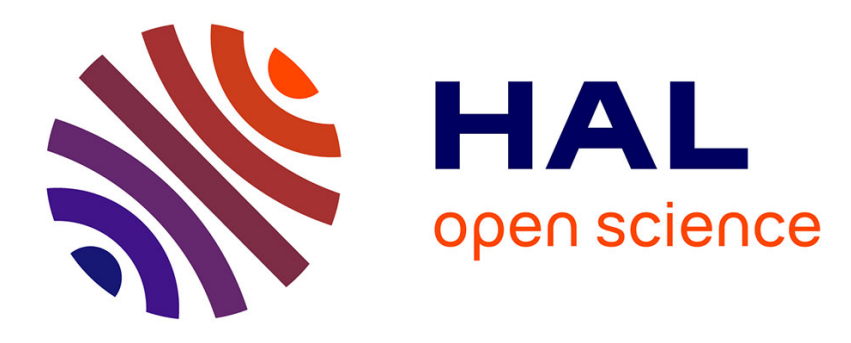

\title{
Étude des deutons et des tritons émis lors du bombardement de noyaux de carbone par des protons de $155 \mathrm{MeV}$ \\ P. Radvanyi, J. Génin
}

\section{- To cite this version:}

P. Radvanyi, J. Génin. Étude des deutons et des tritons émis lors du bombardement de noyaux de carbone par des protons de $155 \mathrm{MeV}$. J. Phys. Radium, 1960, 21 (5), pp.322-325. 10.1051/jphysrad:01960002105032201 . jpa-00236248

\section{HAL Id: jpa-00236248 \\ https://hal.science/jpa-00236248}

Submitted on 1 Jan 1960

HAL is a multi-disciplinary open access archive for the deposit and dissemination of scientific research documents, whether they are published or not. The documents may come from teaching and research institutions in France or abroad, or from public or private research centers.
L'archive ouverte pluridisciplinaire $\mathbf{H A L}$, est destinée au dépôt et à la diffusion de documents scientifiques de niveau recherche, publiés ou non, émanant des établissements d'enseignement et de recherche français ou étrangers, des laboratoires publics ou privés. 


\title{
ETUDE DES DEUTONS ET DES TRITONS \\ EMIS LORS DU BOMBARDEMENT DE NOYAUX DE CARBONE PAR DES PROTONS DE $155 \mathrm{MeV}$.
}

\author{
Par P. RADVANYI et J. GÉNIN, \\ Laboratoire de Physique Nucléaire, Faculté des Sciences, Orsay.
}

\begin{abstract}
Résumé. - On a mesuré les spectres d'énergie des deutons et des tritons émis à $15^{\circ}, 30^{\circ}, 60^{\circ}$ et $120^{\circ}$, lors du bombardement de noyaux de carbone par des protons de $155 \mathrm{MeV}$. Ces mesures ont été faites à l'aide d'un télescope donnant l'énergie et la nature des particules chargées qui le traversent, grâce à la détermination simultanée de leur ionisation spécifique et de leur énergie totale. Les spectres d'énergie obtenus pour les deutons et les tritons - entre $30 \mathrm{MeV}$ environ et l'énergie maximum - sont comparés aux spectres de protons mesurés dans les mêmes conditions. Une grande partie des résultats pourrait être expliquée par des processus de pick-up.
\end{abstract}

\begin{abstract}
The energy spectra of deuterons and tritons emitted at $15^{\circ}, 30^{\circ}, 60^{\circ}$ and $120^{\circ}$ laboratory angles from carbon bombarded by $155 \mathrm{MeV}$ protons were measured. These measurements were made with a scintillator telescope giving the kind and the energy of a charged particle by simultaneous determination of $i t s ~ d E / \mathrm{d} x$ and $E$. The resulting energy spectra for deuterons and tritons - between about $30 \mathrm{MeV}$ and maximum energy - are compared with the proton spectra obtained in the same conditions. An important part of the results could be explained by pick-up reactions.
\end{abstract}

On sait depuis quelques années que les noyaux, sous l'action de protons ou de neutrons de grande énergie, émettent des deutons en proportion relativement notable. Ceux-ci sont émis préférentiel- lement vers l'avant et peuvent avoir une grande énergie, laissant le noyau résiduel dans son état fondamental ou dans un de ses premiers états excités. Les études de ce processus ont été réalisées 
notamment à $90 \mathrm{MeV}$ [1], $95 \mathrm{MeV}$ [2] et $300 \mathrm{MeV}$ [3]. L'émission de tritons énergiques a également été observée. La formation de ces deutons a été interprétée comme le résultat d'un mécanisme de pick-up se produisant soit directement : le proton incident emmenant avec lui un des neutrons du noyau $[4,5,6]$, soit indirectement à la suite d'un premier choc du proton incident dans le noyau [7]. A $675 \mathrm{MeV}$, l'émission de deutons a été considérée comme due à l'éjection de quasideutons hors du noyau [8,9].

De telles expériences doivent fournir des renseignements sur le mécanisme des réactions nucléaires en cause, ainsi que sur la structure des noyaux : quantités de mouvements des nucléons " cueillis ", sous-structures, surface du noyau par des études sur des noyaux de divers $Z$ (le deuton émis - particule peu liée - se formerait à la surface du noyau).

Nous avons voulu étendre ces résultats, en particulier du point de vue de l'extension du domaine d'énergie des deutons émis et de la détermination des spectres de tritons, en utilisant le faisceau externe de protons de $155 \mathrm{MeV}$ du synchrocyclotron d'Orsay. Nos premières expériences ont porté sur le carbone.

Pour étudier, à différents angles par rapport à la direction des protons incidents, les spectres d'énergie des protons, deutons et tritons, nous avons réalisé un télescope permettant de déterminer à la fois l'énergie et la nature des particules chargées émises par la mesure simultanée de leur perte d'énergie spécifique $\mathrm{d} E / \mathrm{d} x$, et de leur énergie totale $E$. Ce télescope, représenté schématiquement sur la figure 1, comporte 3 scintillateurs : le pre-

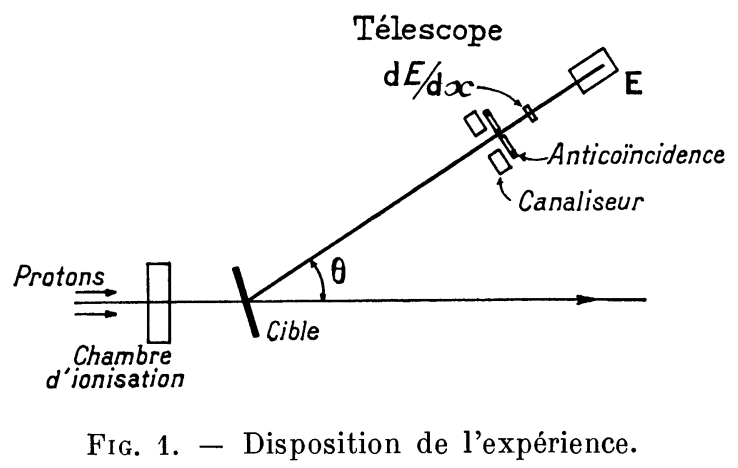

mier est un disque de plastique percé d'un trou de diamètre $10 \mathrm{~mm}$; placé à $70 \mathrm{~cm}$ de la cible, il définit par anticoïncidence l'angle solide vu par le télescope. Le second détecteur est un cristal de CsI(Tl) d'épaisseur $1 \mathrm{~mm}$, permettant de mesurer la quantité $\mathrm{d} E / \mathrm{d} x$ : trop mince, ce cristal donnerait des fluctuations de perte d'énergie trop importantes, trop épais, il conduirait à des énergies limites inférieures trop élevées pour les particules étudiées. Le troisième scintillateur, qui mesure l'énergie totale résiduelle, est un gros cristal de CsI(Tl) de longueur $74 \mathrm{~mm}$, permettant d'absorber complètement les protons les plus énergiques. Les photomultiplicateurs utilisés sont des 53 AVP.

Avec un analyseur d'impulsion à 256 canaux, on mesure le spectre des impulsions $\mathrm{d} E / \mathrm{d} x$ correspondant à une bande d'énergie (environ $4 \mathrm{MeV}$ ) à une énergie donnée $E$ dans le dernier scintillateur. Cette bande est déterminée par un sélecteur à un canal. Les coïncidences (dans un temps de résolution de $1 \mu \mathrm{s}$ ) entre les impulsions provenant de ce sélecteur et celles de la voie $\mathrm{d} E / \mathrm{d} x$ - en anticoïncidence avec les impulsions du plastique de définition - donnent les impulsions de déblocage du 256 canaux.

A chaque angle, on détermine les spectres $\mathrm{d} E / \mathrm{d} x$ correspondant à des bandes prises à une vingtaine d'énergies différentes. Les spectres $\mathrm{d} E / \mathrm{d} x$ donnent ainsi pour chaque énergie résiduelle considérée le spectre de masse des particules chargées traversant le télescope. De cette manière, on sépare correctement les protons, deutons et tritons; quelques ${ }^{3} \mathrm{He}$ et particules $\alpha$ de grande énergie apparaissent également (fig. 2). L'émission de ces deux derniers

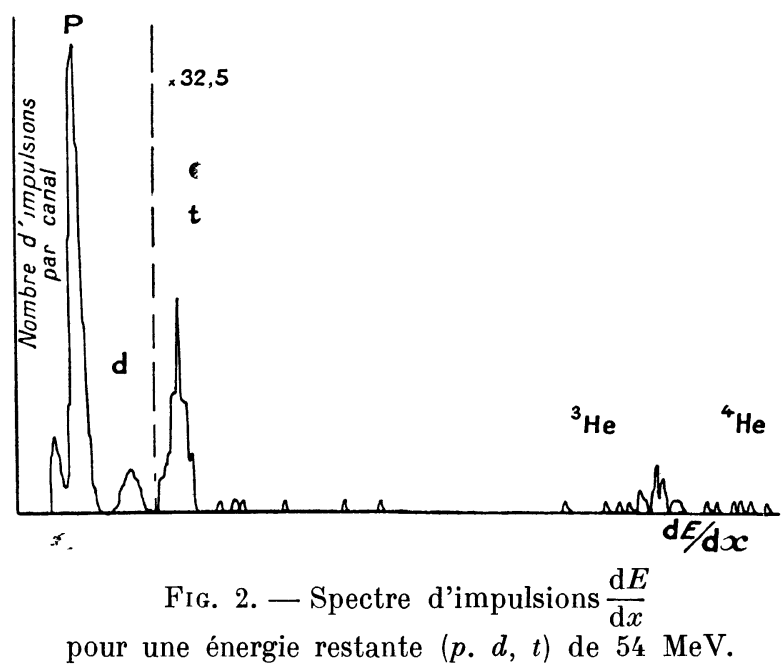

types de particules fera l'objet d'une étude ultérieure.

Nous avons admis que la réponse lumineuse $\mathrm{du} \mathrm{CsI}(\mathrm{Tl})$ aux protons, deutons et tritons de grande énergie était la même, ce qui semble bien vérifié, à 2 ou $3 \%$ près, par la position du pic des deutons de pick-up direct. L'intensité du faisceau de protons est fixée de manière à ne pas dépasser des taux de perte acceptables pour l'anticoïncidence et le 256 canaux.

Les spectres $\mathrm{d} E / \mathrm{d} x$ sont ensuite planimétrés et on remonte, pour chaque type de particule, à l'énergie de départ au milieu de la cible de carbone $\left(0,173 \mathrm{~g} / \mathrm{cm}^{2}\right)$. Les intensités obtenues sont corri- 
gées des pertes électroniques ainsi que des pertes par processus inélastiques (réactions nucléaires) dans le gros cristal de $\mathrm{CsI}(\mathrm{Tl})$. Cette dernière correction, qui a été faite soit graphiquement, soit par le calcul, est particulièrement importante aux petits angles, où les spectres d'énergie des protons et deutons présentent un pic important à l'énergie

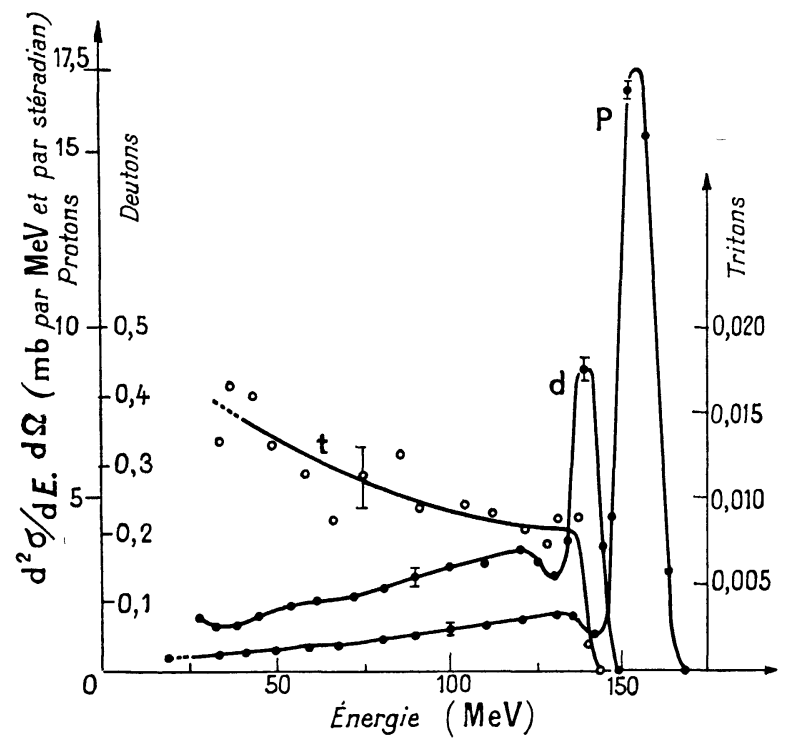

FIg. 3. - Spectres d'énergie des protons, deutons, tritons. Carbone à $15^{\circ}$ (Lab.). $E_{\mathrm{p}}=155 \mathrm{MeV}$.

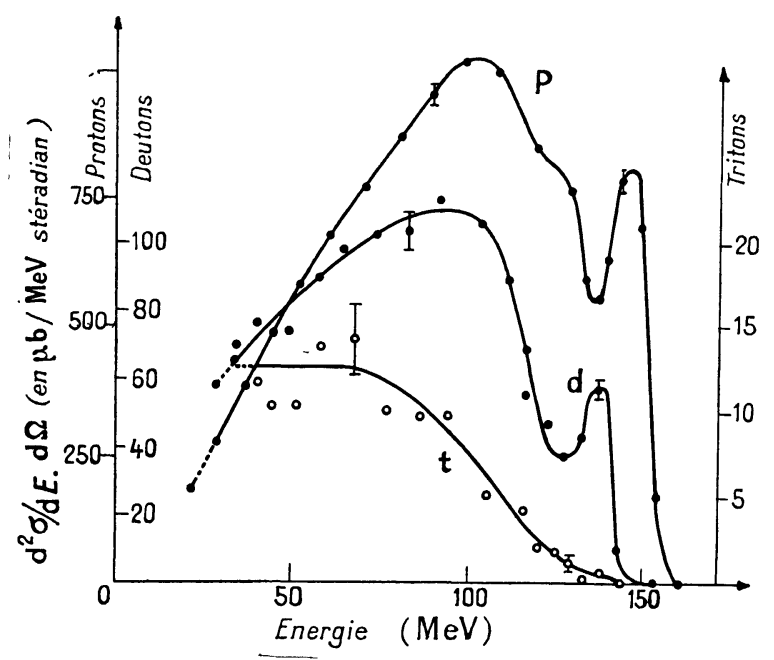

FIG. 4. - Spectres d'énergie des protons, deutons, tritons. Carbone à $30^{\circ}$ (Lab.). $E_{\mathbf{p}}=154 \mathrm{MeV}$.

maximum. Ces phénomènes inélastiques donnent, sur une partie des spectres $\mathrm{d} E / \mathrm{d} x$, un pic à une position inférieure à celle du pic normal de la particule correspondante à l'énergie résiduelle considérée (voir figure 2 à gauche du pic des protons). Une chambre d'ionisation, placée sur le faisceau de protons et étalonnée par comparaison avec un cylindre de Faraday, nous a permis de remonter aux sections efficaces absolues.

Nous avons obtenu ainsi les spectres d'énergie des deutons, des tritons, ainsi que des protons

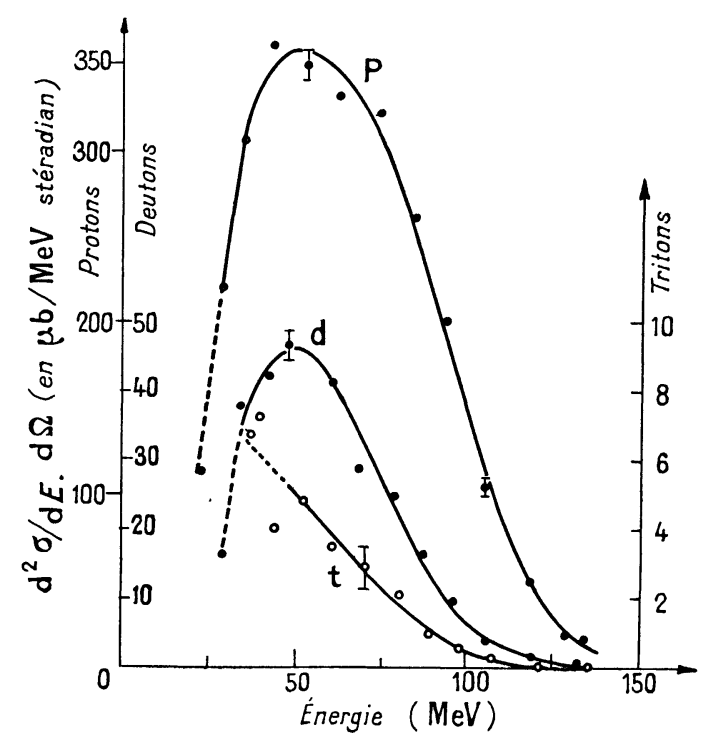

Fig. 5. - Spectres d'énergie des protons, deutons, tritons. Carbone à $60^{\circ}$ (Lab.). $E_{\mathbf{p}}=155 \mathrm{MeV}$.

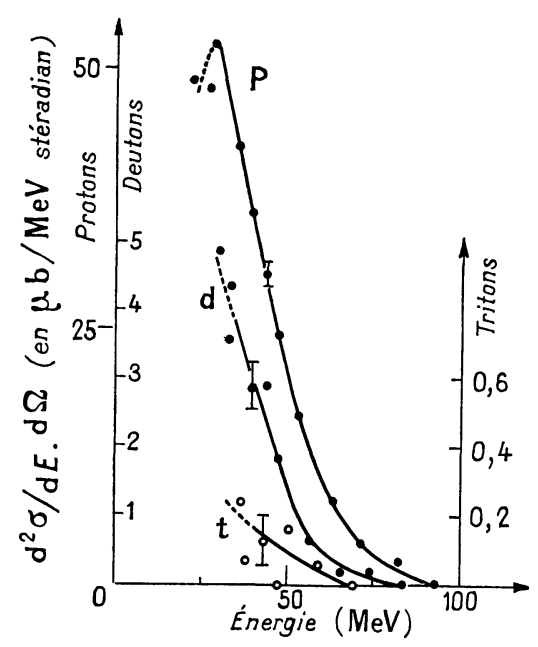

FIG. 6. - Spectres d'énergie des protons, deutons, tritons. Carbone à $120^{\circ}$ (Lab.). $E_{\mathrm{p}}=154 \mathrm{MeV}$.

(mesurés dans les mêmes conditions afin de faciliter les comparaisons) émis par les noyaux cibles de carbone aux angles de $15^{\circ}, 30^{\circ}, 60^{\circ}$ et $120^{\circ}$ (laboratoire) (fig. 3, 4, 5 et 6). La résolution en énergie est médiocre par suite de la largeur de bande et des diverses nécessités de la méthode utilisée pour obtenir une bonne séparation des masses à toutes les énergies. Quelques marges d'erreurs statistiques typiques ont été indiquées sur les courbes. A cause 
de la largeur en énergie de la bande d'analyse au milieu de la cible, l'incertitude sur le dernier point à $15^{\circ}, 30^{\circ}$ et $60^{\circ}$, et sur les deux derniers points à $120^{\circ}$, est nettement plus grande que sur les autres : c'est pourquoi nous avons terminé les spectres d'énergie par des pointillés aux énergies les plus faibles (protons: $\sim 23 \mathrm{MeV}$, deutons : $\sim 29 \mathrm{MeV}$, tritons : $\sim 34 \mathrm{MeV}$ ). L'erreur sur les valeurs des sections efficaces différentielles absolues (en dehors des erreurs statistiques) doit être inférieure à $\pm 15 \%$.

Quelques premières remarques peuvent être faites sur ces spectres :

On distingue nettement, sur les spectres de protons et de deutons à $15^{\circ}$ et à $30^{\circ}$ des pics aux énergies maxima, correspondant à l'état fondamental ou à un des premiers niveaux excités des noyaux résiduels ${ }^{12} \mathrm{C}$ et ${ }^{11} \mathrm{C}$, et des spectres continus audessous des énergies maxima. Les pics de deutons correspondent au processus de pick-up direct. A partir de $60^{\circ}$, on ne distingue plus que des spectres continus. A $30^{\circ}$, le pic de protons doit être constitué par le pic élastique et les pics inélastiques correspondant aux premiers niveaux - non résolus dans notre cas - de ${ }^{12} \mathrm{C}[10]$. Là où la comparaison est possible - et en tenant compte des énergies incidentes différentes - Jes sections efficaces différentielles absolues sont à peu près en accord avec les résultats de Selove [2] et'de Hess et Moyer [3], et en assez bon accord avec des résultats non encore publiés obtenus à Harvard à $145 \mathrm{MeV}$ [11].

Les spectres continus doivent correspondre à des réactions du type $(p, p n),(p, 2 p)$ etc... pour les protons, $(p, d p),(p, d n)$, etc... pour les deutons. Ils présentent un maximum bien apparent jusqu'à $60^{\circ}$. L'énergie du maximum et l'énergie moyenne du spectre continu diminuent quand l'angle augmente. La forme des spectres varie d'un angle à l'autre.

La ressemblance entre les spectres de protons et de deutons à tous les angles est particulièrement frappante : cette analogie semble montrer que les spectres continus de deutons sont dûs en grande partie à un mécanisme de pick-up indirect (pick-up après diffusion).
Aux erreurs statistiques près, les spectres de tritons semblent avoir une forme différente des spectres de deutons, mais on observe aussi à $15^{\circ}$ et à $30^{\circ}$ des tritons ayant l'énergie maximum, et correspondant donc à l'état fondamental de ${ }^{10} \mathrm{C}$, ou à un ou plusieurs de ses pramiers niveaux excités ; au-dessous de cette énergie maximum s'étend un spectre continu de tritons. Trois mécanismes semblent possibles a priori pour la formation de tritons : le pick-up par le proton incident ou par un nucléon diffusé d'une paire de neutrons - ou d'une paire proton-neutron - en corrélation, le pick-up d'un premier neutron, puis d'un second, en deux étapes, et enfin l'éjection d'un « quasitriton ". Le premier de ces mécanismes est sans doute le plus probable.

Il est intéressant de constater que l'on observe encore des deutons et des tritons - bien que beaucoup moins intenses - émis vers l'arrière, à $120^{\circ}$. Peut-être y a-t-il dans ce cas un maximum du spectre de protons vers $29 \mathrm{MeV}$, mais l'incertitude sur les deux derniers points du spectre est trop grande pour pouvoir l'affirmer.

$\mathrm{Au}$ point de vue distribution angulaire, l'intensité des composantes de pick-up direct des spectres de deutons et de tritons diminue rapidement quand l'angle augmente; par contre, l'intensité des spectres continus diminue beaucoup moins vite. Si on compare entre eux les intensités des spectres continus (pics exclus) de protons, deutons et tritons au-dessus de $35 \mathrm{MeV}$, on constate que les rapports $p / d$ et $d / t$ sont approximativement égaux entre eux et ne varient pas sensiblement avec l'angle de $15^{\circ}$ à $60^{\circ}(p / d \simeq d / t \simeq 10)$. Ces rapports sont un peu plus élevés à $120^{\circ}$ (autour de 15) mais ceci est peut-être dû seulement à la fixation arbitraire à $35 \mathrm{MeV}$ de la limite inférieure d'énergie qui intervient davantage dans la comparaison à $120^{\circ}$.

Nous tenons à remercier en particulier M. Brissaud et $\mathrm{M}^{\mathrm{me}}$ Gradsztajn pour l'aide qu'ils nous ont apportée dans les mesures et les montages, $M$. Reide et M. Brun pour toute l'aide apportée en électronique, M. David-Boyer pour le travail particulièrement soigné réalisé pour polir et monter les scintillateurs, ainsi que toute l'équipe qui a as suré la conduite du synchrocyclotron.

\section{BIBLIOGRAPHIE}

[1] Hadley (J.) et York (H.), Phys. Rev., 1950, 80, 345.

[2] Selove (W.), Phys. Rev., 1956, 101, 231.

[3] Hess (W. N.) et Moyer (B. J.), Phys. Res., 1956, 101, 337.

[4] Chew (G. F.) et Goldberger (M. L.), Phys. Rev., $1950,77,470$.

[5] Heidmann (J.), Phys. Rev., 1950, 87, 171.

[6] Greider (K. R.), Phys. Rev., 1959, 114, 786.

[7]. Bransden (B. H.), Proc. Phys. Soc. (London), 1952, 65 A, 738.
[8] Azhgirei (L. S.), Vzorov (I. K.), Zrelov (V. P.), Mescheriakov (M. G.), Neganov (B. S.) et ShaBudin (A. F.), J. Exp. Theor. Phys. (S. S. S. R.), 1957, 33, 1185.

[9] Blockintsev (D. I.), J. Exp. Theor. Phys. (S. S. S. R.) 1957, 33, 1295.

[10] Communication à ce colloque de MM. Garron, JACQuemart, Massonet, Riou et Ruhla.

[11] P. F. Cooper et R, Wilson, cités dans [6]. Note sur épreuves : ces résultats viennent de paraître dans Nuclear Physics, 1960, 15, 373. 